\title{
The Isolation of Human Glioblastoma Cells: An Optimised Protocol
}

\author{
Tomaz Velnar ${ }^{1}$, Uros Maver ${ }^{2}$, Roman Bosnjak ${ }^{1}$, Lidija Gradisnik ${ }^{2}$ \\ ${ }^{1}$ Department of Neurosurgery, University Medical Centre Ljubljana, Ljubljana, Slovenia, ${ }^{2}$ Institute of Biomedical Sciences, \\ Medical Faculty, University of Maribor, Maribor, Slovenia
}

Correspondence: tvelnar@hotmail.com; Tel.: + 3861522 3263; Fax.: + 38615222218

Received: 18 May 2020; Accepted: 19 December 2020

\begin{abstract}
Objective. The aim of this study was to establish an optimised protocol for glioblastoma (GBM) cell isolation from brain resection samples, with a high yield and low risk for contamination. Methods. Human GBM cells can be obtained following cranial tumour operations. In sterile conditions, the fragments of viable tissue removed during surgery were collected. The tissue was cut and mechanically coarsely decomposed. The sediment was harvested after centrifugation, the cells were seeded in suspension, and supplemented with a special medium (Advanced DMEM) containing high level nutrients and antibiotics. Results. In an appropriate environment, the isolated cells retained viability and proliferated quickly. Attachments were observed after ten hours, and proliferation after two days. The time to full confluence was about one week. The cells were stable. Under standard culture conditions, cell proliferation and cluster formation were observed. Cell viability was $95 \%$. Conclusion. The protocol described for isolation is easy, quick and affordable, leading to stable GBM cells. The isolation technique provides sufficient quantities of isolated cells that may be used as an important new tool for in vitro research. The availability of this system will permit the study of cell properties, biochemical aspects, and provides the potential of therapeutic candidates for pathological disorders in a well-controlled environment.
\end{abstract}

Key Words: Human Cell Culture • Glioblastoma Cell Isolation • Isolation Protocol • Glioblastoma.

\section{Introduction}

In recent decades, cell models have become increasingly important for the in vitro study of physiological and pathophysiological processes. To explore the mechanisms of neurotrauma, tumorigenesis and neurodegeneration, in vitro organ culture systems with live neural cells are becoming highly appealing $(1,2)$. Under specific conditions, the isolated cells may be maintained outside the body, in an in vitro environment, and included in various cell models. Since the first attempts at tissue and cell culture development in the early 20th century, which included the method of explantation, isolation techniques have greatly improved $(3,4)$. In $v i$ tro cell cultures and functional cell models are thus becoming an essential research tool. Moreover, the employment of cell cultures is becoming widely recognized due to the decreasing tendency towards animal tests and subsequently lower expense (5-7).

As glioblastomas are the most prevalent primary malignant brain tumours in the adult population, glioblastoma cells are in the focus of research $(6,8)$. Glioblastoma is a universally lethal disease with no effective therapy. From the biological point of view, it is a very heterogeneous tumour, displaying all the characteristics of cancer, with substantial variability among patients. One of the clinical hallmarks of glioblastoma is a high degree of invasiveness, which is evident from the extensive infiltration of the tumour into the surrounding brain parenchyma. The malignant glioblastoma cells can cross tissue barriers and invade the neighbouring tissue as individual cells or collectively, by remodelling the extracellular matrix and their cytoskeleton $(6,9)$. The treatment of glioblastoma is aggra- 
vated, and currently includes maximally safe surgical resection, supplemented with radiation therapy and chemotherapy. In spite of combined treatment modalities, the mean overall survival is still only 21 months, depending on many patient- and tumourrelated factors. The mechanisms leading to glioblastoma formation, invasion and treatment are gradually being understood in in vitro conditions and animal models. These results, however, have not yet been translated into clinically significant therapeutic approaches, despite the ongoing progress $(6,8)$. For in vitro research, numerous new and improved methods of isolation have been used and new ones are being developed, in order to expand and optimise the research possibilities $(6,8-10)$.

The preclinical glioblastoma models currently used in experimental practice are classified into three categories: genetically engineered mouse models, xenografts and syngenic murine models. Every model has its own advantages and disadvantages $(11,12)$. The preclinical mouse models, which are genetically engineered, are essential for the development of new therapies for glioblastoma treatment. These are used for analyses of glioblastoma biology, evaluation of new therapeutic strategies, and identification of therapeutic targets. Genetically engineered mouse models harbour mutations in the components of the main signalling pathways that are altered in human glioblastomas. These genetically engineered mouse models display a long latency to tumorigenesis and advanced tumour heterogeneity, which represents a challenge in preclinical drug testing. An orthotopic mouse model of glioblastoma is constructed by transplanting brain tumour cells derived from glioblastoma into the brain of syngeneic mice. This model develops a glioblastoma with similar features to the human disease. These include aggressive invasion of tumour cells and a high degree of vascularisation. It is frequently used for testing the therapeutic agents used in clinical trials for glioblastoma treatment, representing a valuable preclinical system for advancing present therapies, and for testing novel drugs and drug combinations against glioblastomas $(12,13)$.

The xenografts are classified into two categories: I) glioblastoma cell-line xenografts and II) patient-derived xenografts. The former exhibit the advantages of high engraftment and growth rates, but it is doubtful whether glioblastoma cellline xenografts reflect the true biological nature of glioblastomas. The patient-derived xenografts preserve both the histological and genetic features of the primary tumour. As a result, they represent good preclinical models in glioblastoma research. However, they cannot fully reflect the host's antitumor immunity in human glioblastomas (12-14).

\section{Glioblastoma Cell-Line Xenografts}

Commercially available glioblastoma cell lines, including T98G, A172, U87 and U251, are the most common models used both in vitro and in vivo. These cell lines were derived from glioblastoma patients and are cultured in a serum-containing medium and then xenografted into immunodeficient mice. Glioblastoma cell-line xenografts exhibit the advantages of high engraftment and growth rates, reliable tumour growth and progression, and good reproducibility (12-15). The immortalized cell lines can be readily expanded for an unlimited number of passages in vitro, yielding a large number of tumour cells for experimental use (12). On the other hand, glioblastoma cell-line xenografts do not reflect the clinical characteristics of the original patient-derived tumours, and do not exhibit the tumour necrosis, microvascular proliferation, cell invasion and expression of integrin molecules, differing phenotypically and genotipically from the original patient tumours. Since it is possibly that glioblastoma cell-line xenografts do not reflect the true biological nature of the glioblastomas, this may present a disadvantage in preclinical trials $(12,14-16)$.

\section{Patient-Derived Xenografts}

Patient-derived xenografts, on the other hand, have been a recent focus of glioblastoma research and are being used extensively. They are established by injecting glioblastoma tumour spheres produced under serum-free neurosphere-culture conditions, into immunodeficient mice. The tumour spheres 
have some advantages over glioblastoma cell lines in in vitro conditions. These models retain both the genetic and histological features of the primary tumour from which they were derived. Patient-derived xenografts cells are not subject to the stresses that can arise in cell cultures (17-20). Additionally, their molecular profile is stable over time, they are tumorigenic, and the tumour spheres retain the molecular profile, which is similar to that of the patient's original tumour (21-24). The disadvantage is that not all human glioblastomas can be successfully cultured as tumour spheres $(12,25)$.

The aim of the study was to establish a relatively quick and easy protocol for the isolation of glioblastoma cells from brain resection samples, with a high yield and a low risk for contamination.

\section{Materials and Methods}

\section{The Source of Tissue}

The tissue for glioblastoma isolation was obtained from adult patients following elective cranial tumour surgery. The permission for human brain tissue use was obtained from the ethical committee, and written informed consent was acquired from the patients before the experiment. The cells were isolated from samples taken from various tumour parts: I) from the resection margin on the border to macroscopically normal brain tissue, II) from the superficial tumour parts, III) from deep inside the tumour (approximately $1 \mathrm{~cm}$ from the tumour surface) and IV) from the tumour core. Surgically, subtotal resection was performed. Our samples exhibited the typical microscopic and macroscopic glioblastoma features: necrosis, hypercellularity, nuclear atypia, haemorrhages, thrombosed tumour vessels and vascular proliferation. Altogether, there were five donor patients. In every experiment and for every patient-donated tissue, the isolation procedure was the same as described. A fresh-frozen section during the operation confirmed the tissue as glioblastoma, WHO IV. The resected tissue specimen was examined in the operating theatre, and some of the viable tumour tissue was used for the isolation. The necrotic and haemorrhagic parts were not used for cell isolation and these were macroscopically removed during the tissue preparation in the operating theatre. The sample size of the tumour tissue varied between 2 and $3 \mathrm{~cm}^{3}$. These tissue specimens corresponded to the cortical and subcortical regions. In sterile conditions, fragments of viable tissue removed during the operation were collected, stored in $20 \mathrm{ml}$ of Advanced DMEM medium, supplemented with $100 \mathrm{IU} / \mathrm{ml}$ penicillin, 0.1 $\mathrm{mg} / \mathrm{ml}$ streptomycin, $2 \mathrm{mM}$ L-glutamine, and immediately taken to the laboratory.

\section{Reagents}

All materials and chemicals used were of laboratory grade. The advanced DMEM cell culture medium was purchased from Thermo Fisher Scientific (Waltham, Massachussets, USA). The heat inactivated foetal bovine serum was acquired from Gibco (by Thermo Fisher Scientific, Waltham, Massachussets, USA). The penicillin, streptomycin, L-glutamine, phosphate-buffered saline (PBS) and trypsin/EDTA were procured from SigmaAldrich (Merck KGaA, Darmstadt, Germany). All other chemicals were obtained from common commercial suppliers.-

\section{Preparation of Tissue for the Cell Culture}

The experiment was performed in a $2^{\text {nd }}$ degree biosafety level cell laboratory. After delivery, the tissue fragments were stored in PBS containing $100 \mathrm{U} / \mathrm{ml}$ penicillin and $0.1 \mathrm{mg} / \mathrm{ml}$ streptomycin. In sterile conditions, the tissue specimen was examined again. The macroscopically necrotic parts were discarded. The blood was removed by washing the sample with the saline and trypsin/EDTA, and centrifugation, where the coarse parts and contaminates were removed. The viable part of the tumour was washed three times with PBS. The PBS was decanted and $0.25 \%$ trypsin/EDTA was added. The tissue was submerged in trypsin/EDTA throughout, in order to prevent desiccation. The tissue was then cut into small pieces $\left(<1 \mathrm{~mm}^{3}\right)$ to achieve coarse mechanical decomposition. After one-hour incubation in a controlled atmosphere 
at $37^{\circ} \mathrm{C}, 5 \% \mathrm{CO}_{2}$, the Advanced DMEM medium was added and the suspension was transferred into centrifuge tubes. Centrifugation at 200G-force for 5 minutes followed. The sediment was resuspended in $14 \mathrm{ml}$ of cell culture medium, containing penicillin (100 IU/ml), streptomycin $(0.1 \mathrm{mg} / \mathrm{ml})$, L-glutamine $(2 \mathrm{mM})$ and 5\% FBS, and then plated in two T25 tissue culture flasks. The resulting cell suspension was incubated for two weeks at $37^{\circ} \mathrm{C}$ in $5 \% \mathrm{CO}_{2}$ leading to the preferential proliferation and survival of glioblastoma cells. The medium was replaced twice weekly.

\section{The Culture of Primary Glioblastoma Cells}

Primary glioblastoma cells were routinely cultured in T25 flasks and incubated at $37^{\circ} \mathrm{C}$ in a controlled atmosphere with $5 \% \mathrm{CO}_{2}$. The density for cell culturing was 800000 cells for T25 flasks. After one week in the culture, they became $100 \%$ confluent and were split in a 1:3 ratio with $0.25 \%$ trypsinEDTA. This was followed by centrifugation at 200G-force for 5 minutes. The cell sediment was resuspended in $21 \mathrm{ml}$ of fresh medium with 5\% FBS. The cell suspension was transferred to three T25 flasks. The cultures were then incubated, and growth was monitored. In this way, the cell culture of the first passage was obtained. The first passage cells were grown for five to seven days, until 100\% confluent. Additionally, some cultures were frozen and then thawed again. The growth was monitored under an inverted microscope.

\section{Results}

The human glioblastoma primary cultures described in the experiment consisted of rapidly growing cells that were isolated from the tumour of adult donors. The primary glioblastoma cultures were $100 \%$ confluent after one week. The cells were then split in a 1:3 ratio and transferred to culture flasks. Attachments were observed after ten hours and marked proliferation followed after two days. After five to seven days, a $90 \%$ confluent culture of the first passage was obtained. We grew the cultures up to the tenth passage. Some of

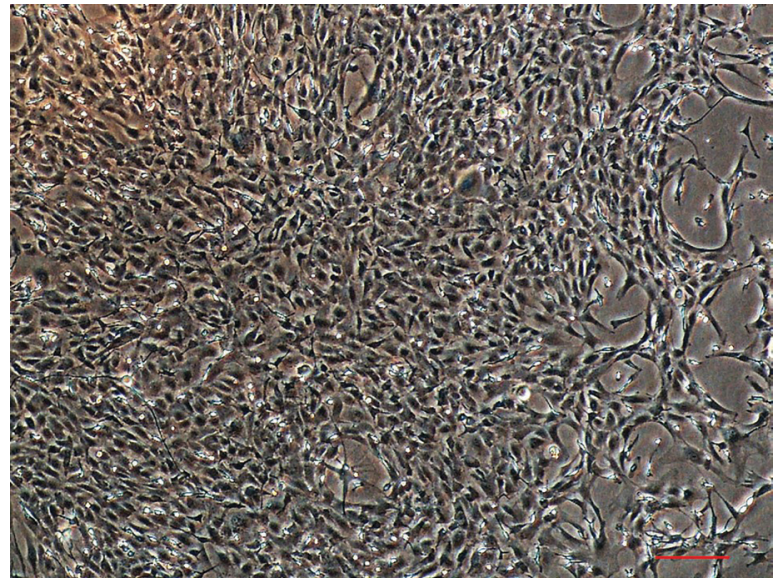

Figure 1. Primary glioblastoma cells. One week after isolation, glioblastoma cells completely cover the surface of the flasks, with the formation of strong intercellular connections (100\% confluence). Images were taken at x40 magnification on a Nicon Diaphot 300 inverted microscope. Scale bar $=100 \mu \mathrm{m}$.

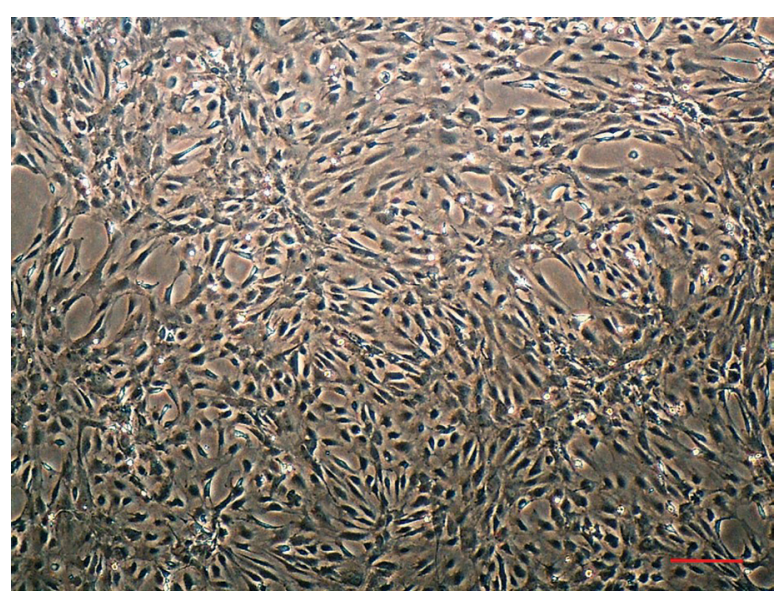

Figure 2. Glioblastoma cell culture in the first passage. One week after the first trypsinization, the glioblastoma culture was $90 \%$ confluent. Images were taken at x40 magnification on a Nicon Diaphot 300 inverted microscope. Scale bar $=100 \mu \mathrm{m}$.

these cells were stored in liquid nitrogen. Viability of $95 \%$ was observed when the cells were thawed and reseeded. These cultures also grew normally after plating (Figure 1 and Figure 2).

We performed this protocol five times, since we had five donor patients. The tissue sources came from glioblastoma patients and were taken during elective surgery. In every experiment and for every patient-donated tissue, the isolation procedure 


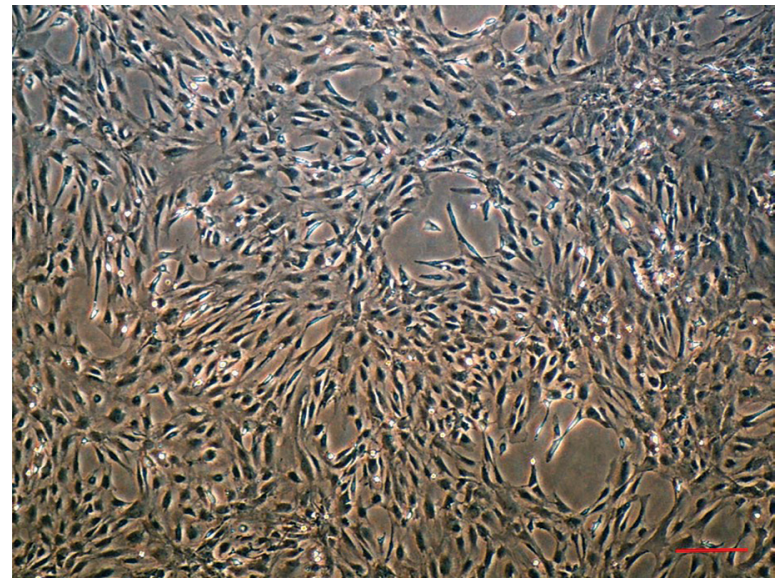

Figure 3. Glioblastoma cell culture in the first passage, isolated from the second patient. One week after the first trypsinisation, the glioblastoma culture was $90 \%$ confluent, as was the case for the first patient. Images were taken at x40 magnification on a Nicon Diaphot 300 inverted microscope. Scale bar $=100 \mu \mathrm{m}$.

was the same as described, leading to the isolation of the glioblastoma cells. The growth characteristics were very similar in all five isolations (Figure 3). According to these results, we think that the method, described in the protocol, was reproducible. Additionally, the cells were frozen and stored in liquid nitrogen, and then thawed and seeded again. These cells also grew normally.

Examination of the morphological properties of these cells showed a distinctive appearance that changed depending on the cell seeding density. The characteristic nucleus shape was polygonal to round, and triangular with scarce cytoplasm. The shape of the cells varied during the attachment process and growth, from round or oval to polygonal, which was characterised during the growth in confluent culture. After 24 hours, most of the cells were attached to the substratum. The shape alterations from round to polygonal were visible at that time. The cells grew well and were easy to maintain in culture, which had a cobblestone appearance, with defined cell borders. The average time to the formation of the confluent culture was one week

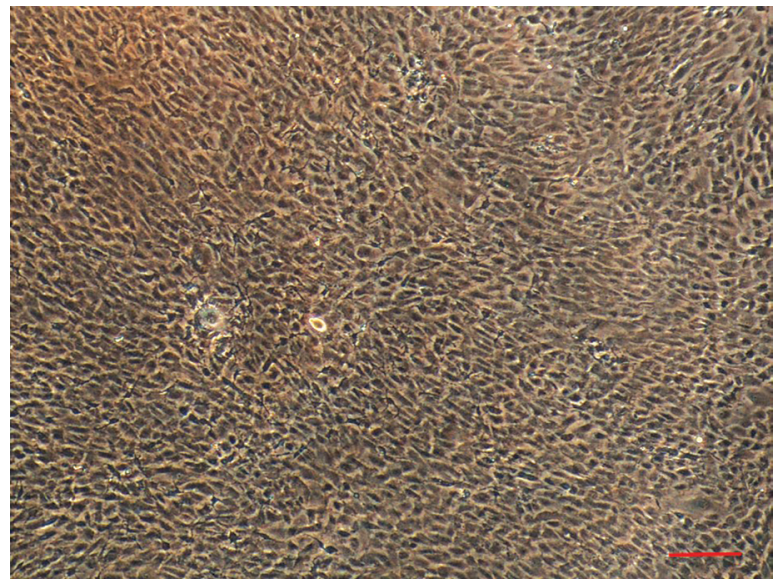

Figure 4. A fully confluent GBM culture. A characteristic polymorphic appearance can be noted. The nuclei display various shapes with scarce cytoplasm. Nicon Diaphot 300 inverted microscope. Scale bar $=100 \mu \mathrm{m}$.

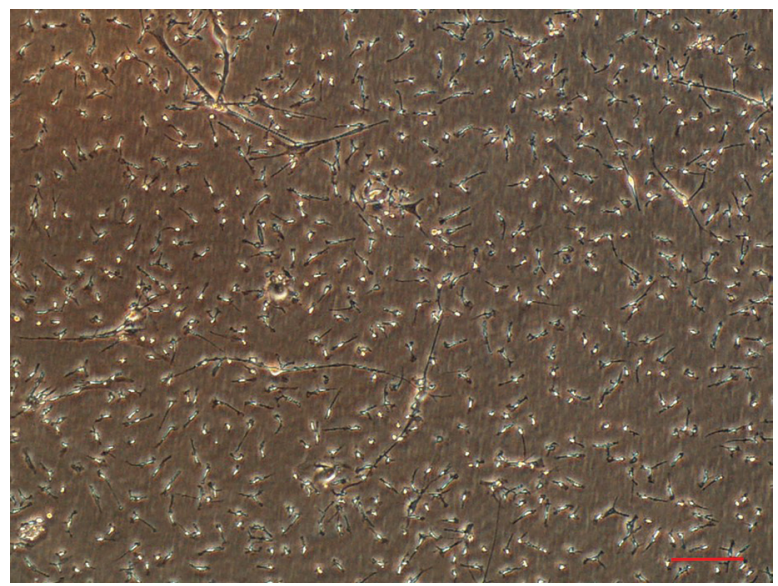

Figure 5. The cells started to attach to the substratum approximately ten hours after seeding. Some cells are still rounded and some exhibit elongated processes. Various cell shapes can be observed. Nicon Diaphot 300 inverted microscope. Scale bar $=100 \mu \mathrm{m}$.

(Figure 4 and Figure 5). Then, the growth did not stop due to the lack of contact inhibition and the cells started to accumulate in multiple layers. This is a typical growth property of cancer cells. Some of the patients' tumour and cell culture characteristics are presented in Table 1. 
Table 1. The Patients'Tumour and Cell Culture Characteristics

\begin{tabular}{lllllllll}
\hline Patient & Diameter & Location & Sample $^{+}$ & Time $^{*}$ & Number $^{\S}$ & Per cent $^{\text {I }}$ & Cellq $^{\text {Cell** }}$ \\
\hline 1 & $4 / 1$ & Right frontotemporal & 3 & 10 & 800000 & 90 & 11 & 48 \\
\hline 2 & $5 / 1$ & Right frontal & 2.5 & 20 & 800000 & 90 & 9 & 52 \\
\hline 3 & $4 / 1.5$ & Right temporal & 2 & 15 & 700000 & 88 & 10 & 54 \\
\hline 4 & $3 / 0.5$ & Right frontal & 2 & 20 & 750000 & 90 & 10 & 48 \\
\hline 5 & $6 / 2$ & Right frontotemporal & 3 & 10 & 850000 & 94 & 9 & 46 \\
\hline
\end{tabular}

*Approximate tumour diameter/necrotic core size $(\mathrm{cm}) ;{ }^{\dagger}$ Sample size $\left(\mathrm{cm}^{3}\right) ;{ }^{\ddagger}$ Approximate time to cell laboratory (min); ${ }^{5}$ Number of cells obtained after dissociation (for a T25 flask); "IPercentage of confluence after one week; "Cell attachment (hours after seeding); **Cll proliferation (hours after seeding).

\section{Discussion}

Glioblastomas are the most common and malignant primary brain tumours in the adult population (9). They are a heterogeneous malignancy, composed of all glial cell types from the central nervous system. In addition to the inter-patient differences in glioblastoma tumours, there is also a well-known intratumoural cellular heterogeneity. This biological heterogeneity is also evident in its resistance to pharmacology, radiation and surgery, and may result in tumour recurrence (26). Since they contain multiple subclonal mutations, this makes them highly adaptable entities, resistant to all therapeutic approaches used today (5, 26-28). It is almost impossible to remove a glioblastoma surgically or treat it completely with radio- and chemotherapy. Despite their resistance to therapy, numerous drugs have shown promising results in preclinical and clinical studies. As a result, primary cultures of glioblastoma cells represent an important target for basic and translational neuroscience research, especially for in vitro cell models $(4,10,29)$.

There have been various reports about glioblastoma cell culture isolation, each with a different technique, and advantages and drawbacks (30-33). We have developed a simplified protocol for enriched primary glioblastoma culture, derived from neurosurgical patients. In the experiment, we used resection specimens from glioblastomas, removed from the right frontotemporal lobes. All samples obtained were excess tumour tissue that corresponded to cortical and subcortical areas. Care was taken to remove the tumour necrosis, and to utilise the vital part of the tumour, which was then used for the culture preparation. This is one of the important steps in establishing a cell culture, since the necrotic parts contain few viable cells and this may lower the cell yield.

The majority of glioblastomas that are removed during surgery are taken for histological examination. We used small resection specimens for the experiment. The tissue was taken from various tumour parts, in order to ensure the most representative samples, since glioblastomas are composed of heterogonous cell populations (22-24). The macroscopically necrotic parts were avoided, since these were not suitable for cell isolation. During the isolation process, it was necessary to develop an effective technique for maintenance of the cell culture. This is often complicated and challenging, and it may take a long time to establish an efficient and reliable culture $(3,32)$. Our protocol was simplified, not involving neurospheres or flow cytometry for cell isolation and the culturing or density separation processes. The tissue was only involved in coarse mechanical degradation and exposed for a short period to trypsin/EDTA in order not to damage the cells to a large extent. Then, immediate plating followed. With a limited degree of tissue degradation and fast culturing, it is possible to obtain a large number of viable cells, allowing the majority of them to attach and proliferate further. We used trypsin for enzymatic digestion, which was necessary in the beginning, when the tissue was brought to the laboratory. We had superior results with trypsin use, since the tissue decomposed better, and the cell yield in the subsequent steps of the isolation process was higher. The unattached contaminant cells were removed in the course of 
culture growth, during medium exchange and gentle washing of adherent cells. Other isolation protocols described so far used more complicated methods for establishing the culture, especially in the tissue decomposition procedure (22, 23, 31-35). With every additional step in isolation, the cells are exposed to potential damage, and thus the cell yield drops. Our cell yield was high, with a 100\% confluency after one week and viability of $95 \%$.

The data were compared to other research protocols, and we saw the following advantages and disadvantages of the described method: I) The tissue source for isolation came from adult donors. This means that the tissue is easily accessible, since there are many more surgical procedures that may provide tissue for experimentation; II) The transport to the laboratory may vary and is usually long in brain samples taken during surgery. They are first brought to the pathology department and the tissue is separated there. The parts are usually taken to the cell laboratory as a secondary priority. This transportation time is typically less than two hours $(36,37)$. In our case, the tissue transportation time was up to 20 minutes. The specimen for the cell laboratory and for pathology examination were already separated in the operating theatre, shortening the time to isolation, and improving the quality of the tissue taken. This resulted in a better cell yield during isolation in the cell laboratory; III) In our protocol we observed that it was not necessary to dissociate the brain tissue to a high extent in order not to lose cells during fine mechanical decomposition. Only coarse mechanical dissociation and a brief period of trypsin addition sufficed, causing less mechanical damage to the cells, thus improving their survival and the subsequent cell yield. It is this process that leads to the high cell survival rate, proliferation and good growth during the isolation procedure; IV) The storage and the transport procedure are extremely important, not only the time needed to the laboratory. In our case, the fragments of viable tissue were collected after surgical resection. They were stored in the Advanced DMEM medium with supplements, and brought to the laboratory immediately; V) The tumour parts chosen for the isolation were carefully selected during surgery. The tissue in our experiment was not taken unselectively from the tumour. We avoided taking the necrotic parts, as these do not add to the cell isolation yield. Only macroscopically viable tissue was taken. $\mathrm{Ne}$ crosis may contaminate the cell suspension during the preparation, and interfere with cell growth during the isolation. Additionally, the contamination risk is higher; VI) Only 5\% FBS was used, as the cells were also successfully isolated with the Advanced DMEM. Other studies used different media and therefore, the addition of $10 \%$ FVS was necessary; VII) Since the tissue was taken from the tumour, the tumorigenic nature of the isolated cells can be confirmed. The samples were taken from the tumour areas verified by neuronavigation and 5-aminolevulinic acid (5-ALA), so any doubt that healthy tissue was obtained was excluded. Additionally, it was surgically evident that the rested tissue was pathologically altered. Again, necrotic pars were excluded.

In the culture, the isolated cells grew rapidly. They were composed of firmly adherent cells of various morphologies. The culture became $90 \%$ confluent after one week, and it was possible to grow cells until the tenth passage. Although we did not cultivate them further, we assume that the cells would grow longer due to their cancer-like characteristics. After plating, attachments were observed after 10 hours, and marked proliferation followed after two days. The time to full confluence was about one week. The cells grew normally even when frozen and thawed again. When observed under a light microscope, the typical characteristics of glioblastoma cells were observed during growth. The cells were pale-staining and polymorphic, displaying round to oval or lobate nuclei, with little visible cytoplasm. The cells were grown in flasks in a single layer and after the formation of a confluent culture, the growth continued, which is characteristic for cancer cells, due to the loss of contact inhibition $(7,35)$. When the confluent layer was formed, the cells began to accumulate in domes, or grew in several layers. Our isolated cells exhibited a high proliferation index, which enabled the rapid formation of a confluent culture. 
Due to their fast growth, the cultures required continuous monitoring of the growth conditions, like other rapidly growing cells.

During the cell isolation procedure, doubt always exists that the cells in culture may not be the target cells. It is true that when preparing the cell isolation from the brain, other contaminant cells may be present, including microglia $(38,39)$. The separation of microglia is done by mechanical separation using the physical properties of these cells $(38,40-44)$. In our case, microglia were removed when changing the medium by washing the attached cells, and removing and discarding the loosely adherent cells. It is possible to reduce the amount of microglia to to $5 \%$ or less, and according to some authors to less than $1 \%(39,45,46)$. The isolation process of glioblastoma cells differs from the isolation of oligodendroglia and astrocytes. It also differs from the isolation of microglia $(36,39,47)$. All these protocols are more complex, and the cells are not so easily established in the culture. In our experiment, we did not observe any additional cell layer over the glioblastoma cells during the culture growth. It is probable that the contaminant cells were removed during the medium change, culture washing and in the subsequent passages. Additionally, glioblastoma cells proliferate faster than healthy oligodendrocytes and astrocytes, and the cells that are a minority in the cell culture are lost from the culture during the growth of other cells. Moreover, glioblastomas are heterogeneous tumours with mixed cell populations. It is believed that there are three cells for the origin of glioblastomas: oligodendrocyte precursor cells, neural stem cells (NSCs) and NSC-derived astrocytes and oligodendrocyte precursor cells (OPCs). In such cases of heterogeneous tumours, the contamination of glioblastoma tumour cells by microenvironmental components, such as macrophages and astrocytes, is also not important, since all the constituents are malignant and neoplastic (48).

In this study, we have described only the protocol from the tissue specimen to the cell culture. This was a new and improved protocol for a highly enriched glioblastoma culture from adult human brains. The isolation technique is quick, easy and cost-effective, and provides sufficient quantities of isolated cells. The cell proliferation rate was rapid, enabling us to reach a $100 \%$ confluent culture after one week. Later on, during the first passage, a $90 \%$ confluent culture was obtained after one week. Viability of $95 \%$ was observed. The protocol by itself is quick and relatively easy, and also the reagents used are of a standard type, which are easily obtainable. An important observation was that the cells did not need supplementation with $10 \%$ FBS, since we used Advanced DMEM. This type of medium was sufficient for the growth conditions. Since this is classified as a less-serum medium, a lower quantity of FBS is needed to supplement the mixture for cell growth. Advanced DMEM is a rich medium by itself, and therefore no additional increments of FBS are needed. When using other types of media for cell experiments, FBS is supplemented according to the protocols employed, the cell needs and the composition of the media used. Additionally, the price of the reagents was advantageous, making the possibilities for isolation processes more accessible. The intention of this article is to present an improved isolation protocol or isolation technique for glioblastoma cells per se. At the time of writing, we have performed this protocol five times, since we had five donor patients. The isolation procedure was the same as described, and the growth characteristics were very similar in all five isolations. According to these results, we believe that the method described in the protocol is reproducible. A very important point that needs to be taken into account in future GBM cell isolation is the need for further genetic and phenotypic characterization of the isolated cells, which was not done in our case.

Examination of the morphological properties of the growing culture illustrated that glioblastoma cells demonstrated a high degree of cellular division and high plasticity. The cells would make a useful model for research into glioblastoma treatment, and may provide a useful basis for in vitro studies. 


\section{Conclusion}

The isolation protocol described is simple, quick and economical, leading to a viable long-term glioblastoma cell culture. The cell culture generated in this manner employs a very simple mediabased culture technique that takes advantage of the adherent properties and proliferative potential of these cells, which makes them appropriate for almost all types of in vitro studies. The availability of an experimental system with glioblastoma cells will permit the study of cell properties, biochemical aspects, and the potential of therapeutic candidates in a well-controlled environment, using a human glioblastoma cell culture.

\section{What Is Already Known on this Topic:}

Glioblastomas are the most prevalent and malignant primary brain tumours in adults and glioblastoma cells are an important focus of research. For this purpose, in vitro functional cell models, employing glioblastoma cells, are highly appealing. Several methods of isolation have been used, and new ones are being developed, in order to expand and optimise the research possibilities.

\section{What this Study Adds:}

We have presented an improved technique for creating a highly enriched glioblastoma culture from adult human glioblastoma patients. The protocol for isolation described is easy, quick and affordable, leading to a stable glioblastoma cell line. Isolated cells may be used as an important new tool for in vitro research.

Authors' Contributions: Conception and design: TV and LG; Acquisition, analysis and interpretation of data: TV and LG; Drafting the article: TV, UM and LG; Revising it critically for important intellectual content: UM, RB and LG; Approved final version of the manuscript: TV, UM, RB and LG.

Conflict of Interest: The authors declare that they have no conflict of interest.

\section{References}

1. Civita P, M Leite D, Pilkington GJ. Pre-Clinical Drug Testing in 2D and 3D Human In Vitro Models of Glioblastoma Incorporating Non-Neoplastic Astrocytes: Tunneling Nano Tubules and Mitochondrial Transfer Modulates Cell Behavior and Therapeutic Response. Int J Mol Sci. 2019;20(23):6017.

2. Gravina GL, Mancini A, Colapietro A, Vitale F, Vetuschi A, Pompili S, et al. The novel CXCR4 antagonist, PRX177561, reduces tumor cell proliferation and accelerates cancer stem cell differentiation in glioblastoma preclinical models. Tumour Biol. 2017;39(6):1010428317695528.
3. Chesnelong C, Restall I, Weiss S. Isolation and Culture of Glioblastoma Brain Tumor Stem Cells. Methods Mol Biol. 2019;1869:11-21.

4. Romaguera-Ros M, Peris-Celda M, Oliver-De La Cruz J, Carrión-Navarro J, Pérez-García A, García-Verdugo JM, et al. Cancer-initiating enriched cell lines from human glioblastoma: preparing for drug discovery assays. Stem Cell Rev Rep. 2012;8(1):288-98.

5. Qazi MA, Vora P, Venugopal C, McFarlane N, Subapanditha MK, Murty NK, et al.A novel stem cell culture model of recurrent glioblastoma. J Neurooncol. 2016;126(1):57-67.

6. Vollmann-Zwerenz A, Leidgens V, Feliciello G, Klein CA, Hau P. Tumor Cell Invasion in Glioblastoma. Int J Mol Sci. 2020;21(6):1932.

7. Gedye C, Ailles L. Isolation and characterization of cancer stem cells in vitro. Methods Mol Biol. 2013;946:181-204.

8. Lukas RV, Wainwright DA, Ladomersky E, Sachdev S, Sonabend AM, Stupp R. Newly Diagnosed Glioblastoma: A Review on Clinical Management. Oncology (Williston Park). 2019;33(3):91-100.

9. Wirsching HG, Galanis E, Weller M. Glioblastoma. Handb Clin Neurol. 2016;134:381-97.

10. Reitman ZJ, Winkler F, Elia AEH. New Directions in the Treatment of Glioblastoma. Semin Neurol. 2018;38(1):50-61.

11. Qazi M, Mann A, van Ommeren R, Venugopal C, McFarlane N, Vora P, et al. Generation of murine xenograft models of brain tumors from primary human tissue for in vivo analysis of the brain tumor-initiating cell. Methods Mol Biol. 2014;1210:37-49.

12. Huszthy PC, Daphu I, Niclou SP, Stieber D, Nigro JM, Sakariassen PO, et al. In vivo models of primary brain tumors: Pitfalls and perspectives. Neuro Oncol. 2012;14(8):979-93.

13. Jeitany M, Pineda JR, Liu Q, Porreca RM, Hoffschir F, Desmaze C, et al. A preclinical mouse model of glioma with an alternative mechanism of telomere maintenance (ALT). Int J Cancer. 2015;136(7):1546-58.

14. Mahesparan R, Read TA, Lund-Johansen M, Skaftnesmo KO, Bjerkvig R, Engebraaten O. Expression of extracellular matrix components in a highly infiltrative in vivo glioma model. Acta Neuropathol. 2003;105(1):49-57.

15. Kijima N, Hosen N, Kagawa N, Hashimoto N, Kinoshita $\mathrm{M}$, Oji Y, et al. Wilms' tumor 1 is involved in tumorigenicity of glioblastoma by regulating cell proliferation and apoptosis. Anticancer Res. 2014;34(1):61-7.

16. Anderson RC, Elder JB, Brown MD, Mandigo CE, Parsa AT, Kim PD, et al. Changes in the immunologic phenotype of human malignant glioma cells after passaging in vitro. Clin Immunol. 2002;102(1):84-95.

17. Jin K, Teng L, Shen Y, He K, Xu Z, Li G. Patient-derived human tumour tissue xenografts in immunodeficient mice: A systematic review. Clin Transl Oncol. 2010;12(7):473-80.

18. Hidalgo M, Amant F, Biankin AV, Budinská E, Byrne AT, Caldas C, et al. Patient-derived xenograft models: An 
emerging platform for translational cancer research. Cancer Discov. 2014;4(9):998-1013.

19. Daniel VC, Marchionni L, Hierman JS, Rhodes JT, Devereux WL, Rudin CM, et al. A primary xenograft model of small-cell lung cancer reveals irreversible changes in gene expression imposed by culture in vitro. Cancer Res. 2009;69(8):3364-73.

20. Fichtner I, Rolff J, Soong R, Hoffmann J, Hammer S, Sommer A, et al. Establishment of patient-derived nonsmall cell lung cancer xenografts as models for the identification of predictive biomarkers. Clin Cancer Res. 2008;14(20):6456-68.

21. Lee J, Kotliarova S, Kotliarov Y, Li A, Su Q, Donin NM, et al. Tumor stem cells derived from glioblastomas cultured in bFGF and EGF more closely mirror the phenotype and genotype of primary tumors than do serum-cultured cell lines. Cancer Cell. 2006;9(5):391-403.

22. Chen R, Nishimura MC, Bumbaca SM, Kharbanda S, Forrest WF, Kasman IM, et al. A hierarchy of self-renewing tumor-initiating cell types in glioblastoma. Cancer Cell. 2010;17(4):362-75.

23. Günther HS, Schmidt NO, Phillips HS, Kemming D, Kharbanda S, Soriano R, et al. Glioblastoma-derived stem cell-enriched cultures form distinct subgroups according to molecular and phenotypic criteria. Oncogene. 2008;27(20):2897-909.

24. Wakimoto H, Mohapatra G, Kanai R, Curry WT Jr, Yip S, Nitta M, et al. Maintenance of primary tumor phenotype and genotype in glioblastoma stem cells. Neuro Oncol. 2012;14(2):132-44.

25. Wan F, Zhang S, Xie R, Gao B, Campos B, Herold-Mende $\mathrm{C}$, et al. The utility and limitations of neurosphere assay, CD133 immunophenotyping and side population assay in glioma stem cell research. Brain Pathol. 2010;20(5):87789.

26. Prager BC, Bhargava S, Mahadev V, Hubert CG, Rich JN. Glioblastoma Stem Cells: Driving Resilience through Chaos. Trends Cancer. 2020;6(3):223-35.

27. Czapski B, Baluszek S, Herold-Mende C, Kaminska B. Clinical and immunological correlates of long term survival in glioblastoma. Contemp Oncol (Pozn). 2018;22(1A):81-5.

28. Perus LJM, Walsh LA. Microenvironmental Heterogeneity in Brain Malignancies. Front Immunol. 20191;10:2294.

29. Klekner Á, Szivos L, Virga J, Árkosy P, Bognár L, Birkó Z, et al. Significance of liquid biopsy in glioblastoma - A review. J Biotechnol. 2019;298:82-7.

30. Alinezhadbalalami N, Douglas TA, Balani N, Verbridge SS, Davalos RV. The feasibility of using dielectrophoresis for isolation of glioblastoma subpopulations with increased stemness. Electrophoresis. 2019;40(18-19):2592-600.

31. Inocencio J, Frenster JD, Placantonakis DG. Isolation of Glioblastoma Stem Cells with Flow Cytometry. Methods Mol Biol. 2018;1741:71-9.
32. Seidel S, Garvalov BK, Acker T. Isolation and culture of primary glioblastoma cells from human tumor specimens. Methods Mol Biol. 2015;1235:263-75.

33. Iacopino F, Angelucci C, Piacentini R, Biamonte F, Mangiola A, Maira G, et al. Isolation of cancer stem cells from three human glioblastoma cell lines: characterization of two selected clones. PLoS One. 2014;9(8):105166.

34. Azari H, Millette S, Ansari S, Rahman M, Deleyrolle LP, Reynolds BA. Isolation and expansion of human glioblastoma multiforme tumor cells using the neurosphere assay. J Vis Exp. 2011;(56):3633.

35. Brehar FM, Bleotu C, Stefan LM, Buzgariu W, Chivu M, Utoiu E, et al. Isolation and partial characterization of a new human glioblastoma cell line. Chirurgia (Bucur). 2009;104(4):453-61.

36. Jakovcevski I, Filipovic R, Mo Z, Rakic S. Oligodendrocyte development and the onset of myelination in the human fetal brain. Front Neuroanat. 2009;3:5.

37. Rustenhoven J, Park TI, Schweder P. Isolation of highly enriched primary human microglia for functional studies. Sci Rep. 2016;6:19371.

38. Chew LJ, DeBoy CA, Senatorov VV Jr. Finding degrees of separation: experimental approaches for astroglial and oligodendroglial cell isolation and genetic targeting. J Neurosci Methods. 2014;236:125-47.

39. Giffard RG, Ouyang YB. Cell Culture: Primary neural cells. In: Squire LR, editor. Encyclopaedia of neuroscience. Oxford: Academic Press; 2009. p. 633-7.

40. Sharif A, Prevot V. Isolation and culture of human astrocytes. Methods Mol Biol. 2012;814:137-51.

41. Oberheim NA, Takano T, Han X. Uniquely hominid features of adult human astrocytes. J Neurosci. 2009;29(10):3276-87.

42. Montgomery DL. Astrocytes: form, functions, and roles in disease. Vet Pathol. 1994;31(2):145-67.

43. Condic M, Oberstein TJ, Herrmann M. N-truncation and pyroglutaminylation enhances the opsonizing capacity of $\mathrm{A} \beta$-peptides and facilitates phagocytosis by macrophages and microglia. Brain Behav Immun. 2014;41:116-25.

44. Agalave NM, Lane BT, Mody PH. Isolation, culture, and downstream characterization of primary microglia and astrocytes from adult rodent brain and spinal cord. J Neurosci Methods. 2020:108742.

45. Uliasz TF, Hamby ME, Jackman NA. Generation of primary astrocyte cultures devoid of contaminating microglia. Methods Mol Biol. 2012;814:61-79.

46. Welser JV, Milner R. Derivation of microglia-free astrocyte cultures from neural stem cells. Methods Mol Biol. 2012;814:81-91.

47. Goldman SA, Kuypers NJ. How to make an oligodendrocyte. Development. 2015;142(23):3983-95.

48. Yao M, Li S, Wu X, Diao S, Zhang G, He H, et al. Cellular origin of glioblastoma and its implication in precision therapy. Cell Mol Immunol. 2018;15(8):737-9. 